\title{
Gamma Ray Doses in Water Around Sea Floor Hydrothermal Area in the Southern Mariana Trough
}

\author{
Shin Toyoda, Debabrata Banerjee, Hidenori Kumagai, Junichi Miyazaki, \\ Jun-ichiro Ishibashi, Nobutatsu Mochizuki, and Shigeaki Kojima
}

\begin{abstract}
The dose rates in sea water in the Southern Mariana Trough sea floor hydrothermal area were investigated. The dose rate in sea water was less than detection limit (about $0.1 \mathrm{mGy} / \mathrm{year}$ ) near the sea surface while it was around $2 \mathrm{mGy} / \mathrm{year}$ at the sea floor of hydrothermal area, where those levels of dose rates will be negligible for ESR (electron spin resonance) dating of barite of sulfide deposits. During the dive by a submarine vehicle, there were three locations at which the level of dose rate increases more than ten times as much as the usual level. One was very close to a hydrothermal vent, but no hydrothermal activities were found near the other locations.
\end{abstract}

Keywords

Gamma ray $•$ Hydrothermal water $• \mathrm{NaI}$ spectrometer

\subsection{Introduction}

Radioactivity in sea water at sea floor hydrothermal area is one of the important factors for trapped electron dating methods such as by electron spin resonance (ESR) (e.g., Takamasa et al. 2013), as it possibly gives doses, hence additional trapped electrons, to the minerals for which analysis is made to obtain ages. Radioactivity in sea water would also be an indicator of hydrothermal activities as the source

S. Toyoda $(\bowtie)$

Okayama University of Science, Okayama, Japan

e-mail: toyoda@dap.ous.ac.jp

D. Banerjee

Physical Research Laboratory, Ahmedabad, India

H. Kumagai • J. Miyazaki

JAMSTEC, Yokosuka, Japan

J.-i. Ishibashi

Kyushu University, Fukuoka, Japan

N. Mochizuki

Kumamoto University, Kumamoto, Japan

S. Kojima

Graduate School of Frontier Sciences, The University of Tokyo,

Chiba, Japan in sea water of hydrothermal area is dissolved radioactive radium and radon emanated from hydrothermal vents (Kumagai et al. 2012).

Commercial gamma ray spectrometers are not suitable for such studies as they are neither water nor pressure proof. A NaI gamma ray spectrometer was specially designed for deep sea radioactivity (Hattori et al. 2000) and it was used extensively for studies to map the radiation intensities at the sea floor (Hattori and Okano 2001, 2002). In this Chapter, this $\mathrm{NaI}$ spectrometer was used to investigate the radioactivity in the sea water at the sea floor around hydrothermal active area. After making calibration for the spectrometer and examining the shielding by the water, the level of radioactivity of the sea water in the South Mariana Trough hydrothermal area was measured. The radioactivity was also continuously monitored during the dives to see the correlation of the radioactivity with the hydrothermal activity.

\subsection{Calibration of the Nal Spectrometer}

An NaI gamma ray spectrometer was used in the present study, which is designed for deep sea radioactive research. The detector and the preamplifier are enclosed in 
a high-pressure-proof tube of $12 \mathrm{~cm}$ in diameter and $60 \mathrm{~cm}$ in length. It is wired to the submersible vehicle, SHINKAI6500, to supply the electricity and to output the spectrum data. A special program for this NaI spectrometer installed in a $\mathrm{PC}$ in the vehicle records the spectrum every minute.

The background dose at the building No.1 of Okayama University of Science was measured by this NaI spectrometer (Shinkai-type) and by Aloka, JSM-102, for $1 \mathrm{~h}$. The averaged doses were, $69.3 \mathrm{nGy} / \mathrm{h}$, corresponding to $0.697 \mathrm{mGy} / \mathrm{year}$, by Shinkai-type spectrometer and $0.761 \mathrm{mGy} /$ year by Aloka, JSM-102, using G(E) function method. It was found that the dose rate values obtained by Shinkai-type spectrometer have to be multiplied by the correction factor of 1.09 .

\subsection{Gamma Ray Attenuation by Sea Water}

Gamma rays are attenuated by materials with the following equation,

$$
\mathrm{I}=I_{0} e^{-\mu x}
$$

where $\mathrm{I}$ is the gamma ray intensity, $\mathrm{I}_{0}$ gamma ray intensity at $\mathrm{x}=0, \mathrm{x}$ the distance from the source, $\mu$ the attenuation coefficient.

Actual gamma ray measurements were performed at the sea floor to examine the attenuation of gamma ray by sea water in the SHINKAI6500 Dive 1228 during cruise YK1011 operated by JAMSTEC in 2010 at the Southern Mariana Trough. A gamma ray spectrum was obtained for $3 \mathrm{~min}$ attaching the end of the spectrometer tube to basalt with the tube vertical to the rock surface at the sea floor. Then the measurements were repeated at a distance, $15 \mathrm{~cm}$, from the rock surface and at $25 \mathrm{~cm}$. After using the program, the dose rate values were obtained being multiplied by the correction factor, as a function of distance as shown in Fig. 46.1. A curve best fit to equation (1) was obtained by the least square method as shown in the figure. The obtained attenuation coefficient, $\mu$, is $0.100 \mathrm{~cm}^{-1}$, being consistent with the literature value of $0.0971 \mathrm{~cm}^{-1}$ (Firestone et al. 1999).

\subsection{Comparison with OSL Dosimeters}

OSL (optically stimulated luminescence) dosimeters made of $\mathrm{Al}_{2} \mathrm{O}_{3}: \mathrm{C}$ manufactured by Nagase Landauer, Ltd. were kept in a titanium tube. Two titanium tubes were placed on sulfide deposit at Archaean Site, the South Mariana Trough, during the cruise YK11-10 operated by JAMSTEC in 2011. They were left there for 29 days and were recovered during YK11-11.

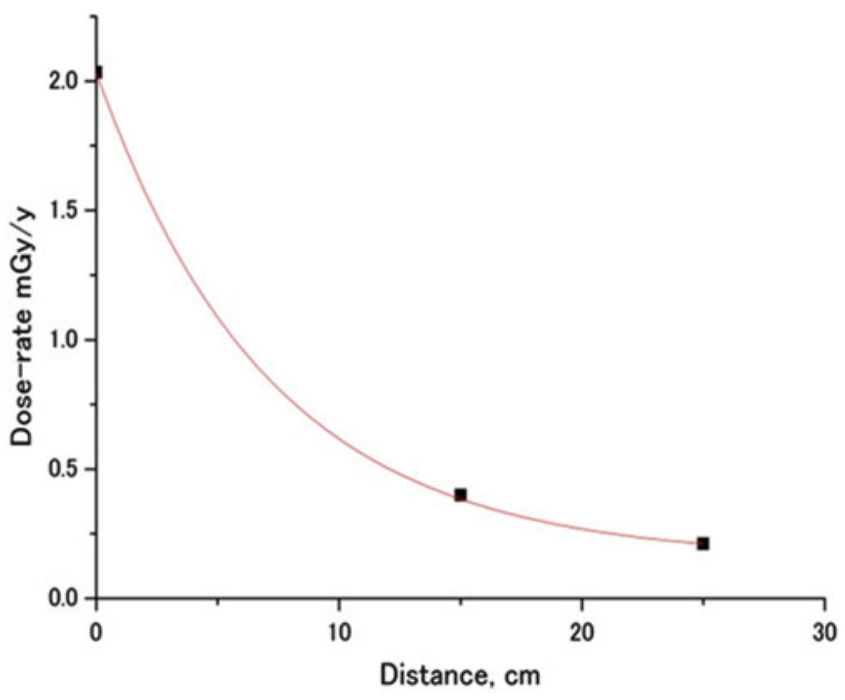

Fig. 46.1 The dose rate observed in water with distance from a basalt surface. The attenuation coefficient was obtained to be $0.100 \mathrm{~cm}^{-1}$

The OSL doses were obtained as the averages of each five specimen. The obtained doses were converted to the dose rates per year to be $1.26 \pm 0.20$ and $0.86 \pm 0.07 \mathrm{mGy} / \mathrm{year}$ for the two tubes located $10 \mathrm{~cm}$ apart from each other. As the attenuation factor of gamma rays in water is $0.100 \mathrm{~cm}^{-1}$ as obtained above, the contribution of the gamma rays in water is drastically reduced beyond $10 \mathrm{~cm}$. The difference in the dose rates, from the two locations $10 \mathrm{~cm}$ apart from each other, would probably be due to the heterogeneity of the doses at the sea floor. The actual gamma ray dose was measured during SHINKAI6500 Dive 1221 by the Shinkaitype $\mathrm{NaI}$ spectrometer with $12 \mathrm{~cm}$ in diameter, at the place same as the titanium tubes were placed with OSL dosimeters inside. The obtained value of $0.92 \mathrm{mGy} / \mathrm{year}$ is consistent with the averaged value by the OSL dosimeters of $1.05 \mathrm{mGy} /$ year within the measurement error.

As results of these calibrating studies, it was shown that this Shinkai-type NaI spectrometer can be used for dosimetry of sea water quantitatively.

\subsection{Survey of Radioactivity in Sea Water at Hydrothermal Area of the South Mariana Trough}

The Shinkai-type NaI spectrometer was placed between the two supports of the submersible vehicle, Shinkai-6500, during the dives, and radioactivity of sea water was continuously monitored. As the position of the spectrometer was at about $80 \mathrm{~cm}$ from the sea floor when the vehicle lands at the floor, the measured doses are of the sea water. The dives, 1223 and 1224 were performed at Archaean site, and 1226 at 


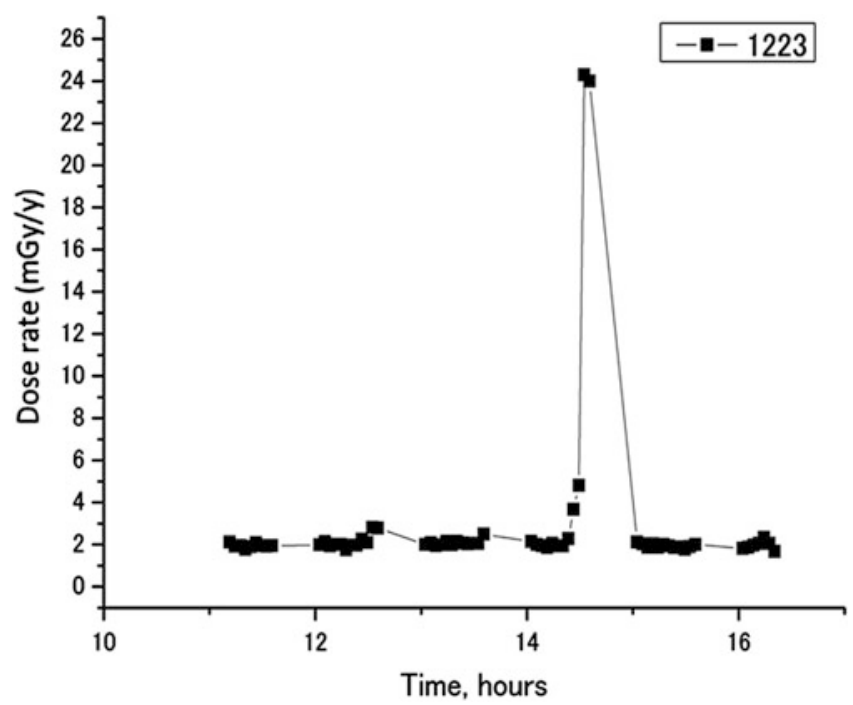

Fig. 46.2 The temporal change of the water radioactivity during the Dive 1223 at Archaean Site. One peak of radioactivity was observed, for which the reason was not found. The horizontal axis denotes the time of the day, while the vertical axis the dose rate in mGy/year

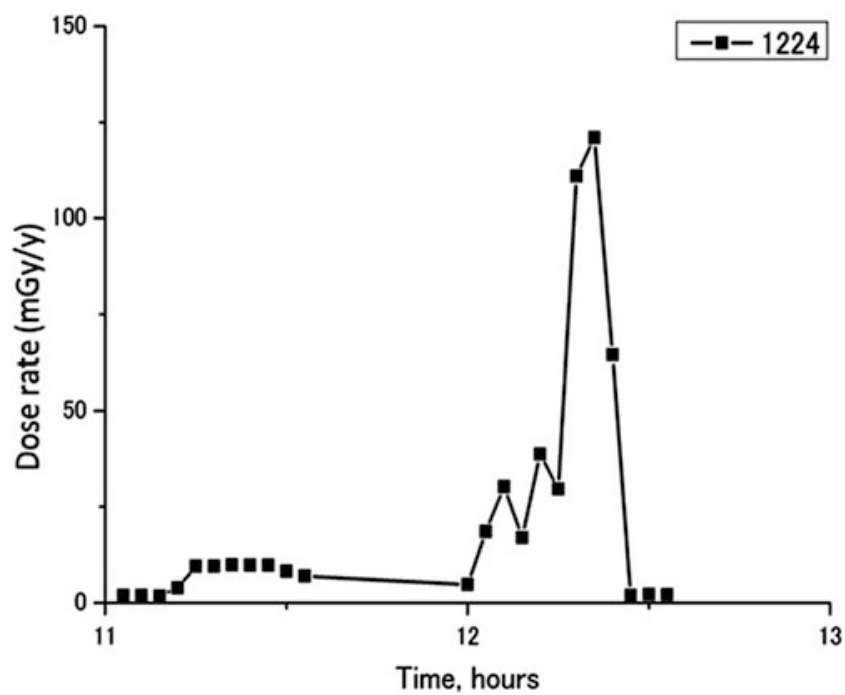

Fig. 46.3 The temporal change of the water radioactivity during the Dive 1224 at Archaean Site. The peaks of radioactivity correspond to the currently active hydrothermal vents. The horizontal axis denotes the time of the day, while the vertical axis the dose rate in mGy/year

Pika site, the Southern Mariana Trough, during the research cruise YK10-11.

The changes of water dose rate were obtained as shown in Figs. 46.2, 46.3, and 46.4. The horizontal axis denotes the time during the day and the vertical axis the dose rate averaged for $5 \mathrm{~min}$ in $\mathrm{mGy} / \mathrm{year}$. The dose rate was below detection limit of the spectrometer (about $0.1 \mathrm{mGy} /$ year) just after the dive starts at the sea surface (Fig. 46.3). The dose rate increased to about $2 \mathrm{mGy} /$ year when the vehicle arrived at the bottom of hydrothermal area, and stayed constant

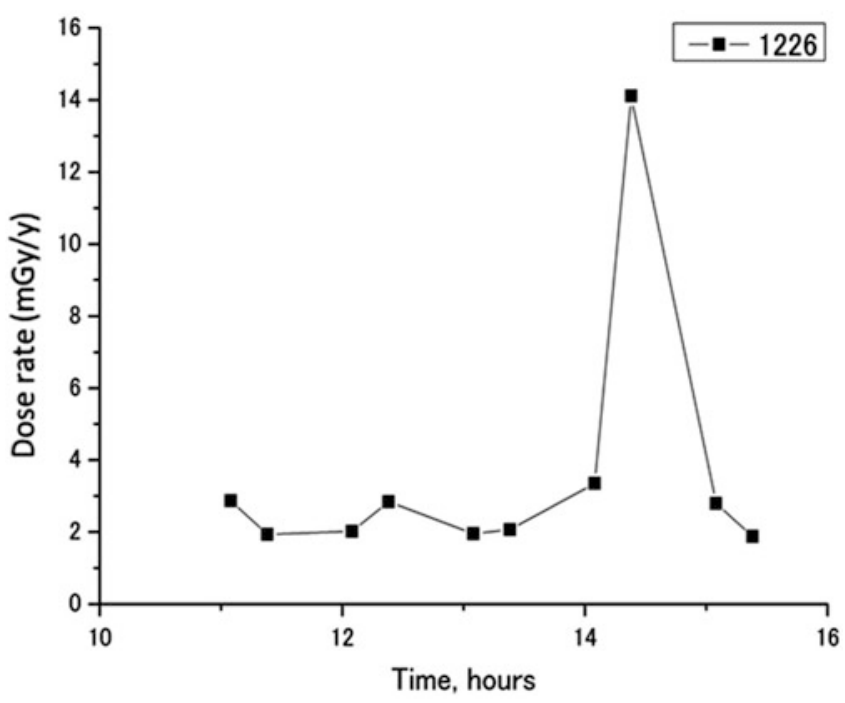

Fig. 46.4 The temporal change of the water radioactivity during the Dive 1226 at Pika Site. One peak of radioactivity was observed, for which the reason was not found. The horizontal axis denotes the time of the day, while the vertical axis the dose rate in mGy/year

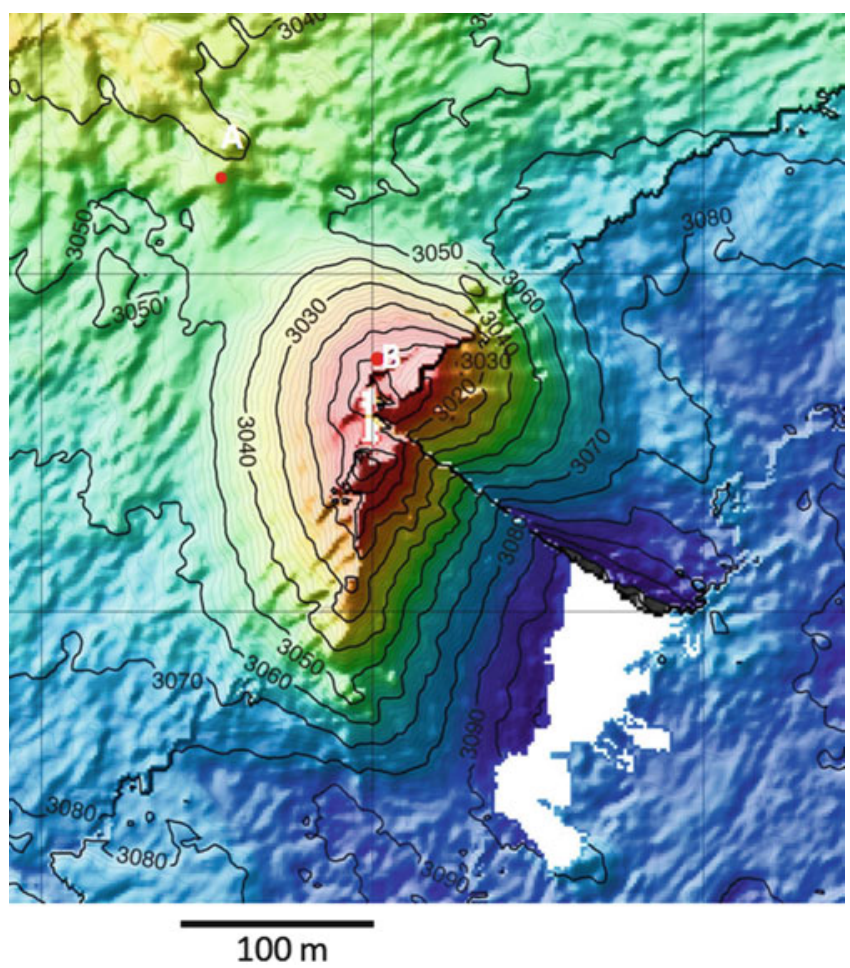

Fig. 46.5 Map of Archaean Site with places where high radioactive water was observed. The location at the top of the rise corresponds to currently active vents (Fig. 46.3), but the reason for higher activity at north-west point (Fig. 46.2) is unknown

during the dive. However, one peak at each dive was observed as shown in Figs. 46.2, 46.3, and 46.4 at locations shown in Figs. 46.5 and 46.6. The peak observed during Dive 1224 (Fig. 46.3) was very high up to $120 \mathrm{mGy} /$ year 


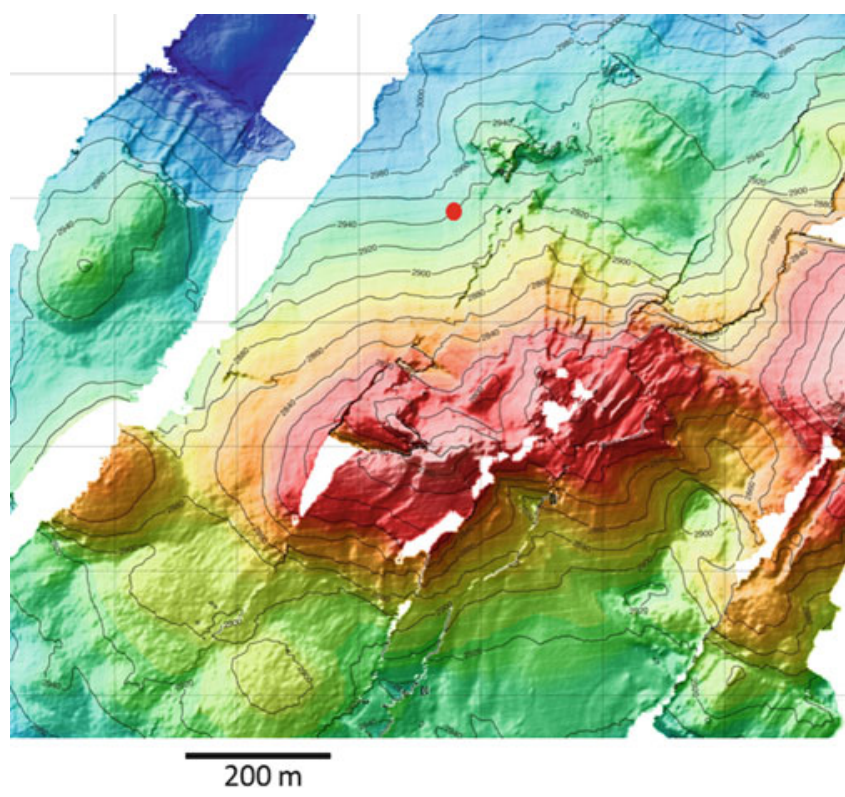

Fig. 46.6 Map of Pika Site with a place where high radioactive water was observed. The reason for this higher activity (Fig. 46.4) is unknown

at the top of the Archaean site (Point B, Fig. 46.5). This was observed when the vehicle stayed at currently active hydrothermal area within about $50 \mathrm{~m}$ in distance. However, the other two peaks (Point A, Figs. 46.5 and 46.6) were observed at the foot of the rise where no currently active hydrothermal activities were found. It was observed within $10 \mathrm{~min}$ (Point A, Fig. 46.5) and 5 min (Fig. 46.6) corresponding to about $20-10 \mathrm{~m}$ in distance. It is possible that these peaks correspond to some unknown currently active hydrothermal vents. If it is the case, another venting can be found by the guide of radioactivity measurements.

The "usual" dose rate of about $2 \mathrm{mGy} /$ year in sea water observed in these sea floor hydrothermal areas is negligible for the dose rate for ESR (electron spin resonance) dating of many barite samples extracted hydrothermal deposits (Fujiwara et al. Chap. 29) as the present dose rate to barite is in the order of several $10 \mathrm{mGy} /$ year.

On the other hand, when the sample is exposed to active hydrothermal vents, the dose rate can be in the order of $100 \mathrm{mGy} /$ year as observed at the three locations in the present study, the sea water dose might have given significant contribution. In this sense, it is recommended to examine the distribution of the dose within the deposit samples. If the dose is higher at the outer part, it is possible that the sample was give high dose rate from the sea water.

\subsection{Summary}

Radioactivity in the sea water in the hydrothermal area in the South Mariana Trough was investigated using a NaI spectrometer designed for use with submersible vehicle. The radioactivity of the surface sea water was below detection limit while the value of the water in hydrothermal area without currently active vents was about $2 \mathrm{mGy} / \mathrm{year}$, which is most probably mixture of water from active vents with high radioactivity and usual sea water. High water radioactivity was found in water close to currently active vents, but also two places without such vents. The dose from water will not affect dating results unless the sample is continuously exposed to active hydrothermal vents.

Acknowledgements The work was supported by TAIGA project, Grant-in-Aid for Scientific Research on innovative Areas (20109004) funded by the Ministry of Education, Culture, Sports, Science and Technology (MEXT). The authors thank Nagase Landauer, Ltd. for providing and for measuring the OSL dosimeters.

Open Access This chapter is distributed under the terms of the Creative Commons Attribution Noncommercial License, which permits any noncommercial use, distribution, and reproduction in any medium, provided the original author(s) and source are credited.

\section{References}

Firestone RB, Shirley VS, Baglin CM, Chu SYF, Zipkin J (1999) Table of isotopes, 8th edn. Willey, USA

Hattori M, Okano M, Togawa O (2000) Sea bottom gamma ray measurement by $\mathrm{NaI}(\mathrm{Tl})$ Scintillation spectrometers installed on manned submersibles, ROV and sea bottom long term observatory. In: Proceedings 2000 International Symposium on Underwater Technology. IEEE Catalog Number 00EX418, pp 212-217

Hattori M, Okano M (2001) New results of sea bottom radioactivity measurement. JAMSTEC J Deep Sea Res 18:1-13 (in Japanese English abstract)

Hattori M, Okano M (2002) Sea bottom gamma ray measurement results of study and modeling of sea bottom radioactive environment. JAMSTEC J Deep Sea Res 20:37-52 (In Japanese w/English abstract)

Kumagai H, Iwase R, Kinoshita M, Machiyama H, Hattori M, and Okano M (2012) Environmental gamma-ray observation in deep sea. In: Feriz Adrovic (eds) Gamma radiation. InTECH, Rijeka, Croatia, ISBN 978-953-51-0316-5, pp 55-74

Takamasa A, Nakai S, Sato F, Toyoda S, Banerjee D, Ishibashi J (2013) $\mathrm{U}-\mathrm{Th}$ radioactive disequilibrium and ESR dating of a baritecontaining sulfide crust from South Mariana Trough. Quater Geochronol 15:38-46 